\title{
Supporting Informantion
}

\section{Twelve-Component Free-Standing Nanoporous High-Entropy Alloys for Multifunctional Electrocatalysis}

Tingting Yu, ${ }^{1}$ Yanyi Zhang, ${ }^{1}$ Yixuan Hu, ${ }^{1}$ Kailong Hu, Xi Lin, Guoqiang Xie, Xingjun

Liu, Kolan Madhav Reddy, * Yoshikazu Ito and Hua-Jun Qiu*

T. Yu, Y. Zhang, K. Hu, X. Lin, G. Xie, X. Liu, H.-J. Qiu

School of Materials Science and Engineering, Harbin Institute of Technology, Shenzhen 518055, China

Email: qiuhuajun@hit.edu.cn

Y. Hu, K. M. Reddy

Frontier Research Center for Materials Structure, School of Materials Science and Engineering, Shanghai Jiao Tong University, Shanghai, 200240, China

Email:kmreddy@sjtu.edu.cn

Y. Ito

Institute of Applied Physics, Graduate School of Pure and Applied Sciences, University of Tsukuba, 1-1-1 Tennodai,

Tsukuba 305-8571, Japan

G. Xie, X. Liu, H.-J. Qiu

Shenzhen R\&D Center for Al-based Hydrogen Hydrolysis Materials, Shenzhen, 518055, China

${ }^{1}$ These authors contribute equally to this work. 


\section{Experimental Section}

\section{Materials synthesis}

Mn-based alloys such as $\mathrm{Mn}_{70} \mathrm{Ni}_{30}, \quad \mathrm{Mn}_{70} \mathrm{Ni}_{15} \mathrm{Cu}_{15}, \quad \mathrm{Mn}_{75} \mathrm{Ni}_{8} \mathrm{Cu}_{8} \mathrm{Co}_{4.5} \mathrm{~V}_{4.5}$, $\mathrm{Mn}_{75} \mathrm{Ni}_{10} \mathrm{Cu}_{10} \mathrm{Co}_{4.5} \mathrm{Pt}_{0.5}, \quad \mathrm{Mn}_{70} \mathrm{Ni}_{7.5} \mathrm{Cu}_{7.5} \mathrm{Co}_{4.2} \mathrm{~V}_{4.2} \mathrm{Fe}_{2} \mathrm{Mo}_{2} \mathrm{Pd}_{0.5} \mathrm{Pt}_{0.5} \mathrm{Au}_{0.5} \mathrm{Ru}_{0.5} \mathrm{Ir}_{0.5}$ and $\mathrm{Mn}_{67.5} \mathrm{Ni}_{7.2} \mathrm{Cu}_{7.2} \mathrm{Co}_{4} \mathrm{~V}_{4} \mathrm{Fe}_{4} \mathrm{Mo}_{0.5} \mathrm{Cr}_{0.5} \mathrm{Pd}_{0.5} \mathrm{Pt}_{1} \mathrm{Au}_{0.5} \mathrm{Ru}_{1} \mathrm{Ir}_{0.5} \mathrm{Ag}_{0.5} \mathrm{Rh}_{0.5} \mathrm{Os}_{0.5} \quad$ (at.\%) were prepared by melting pure melts ( $>99.9$ wt.\%) using an induction-melting furnace under the Ar protection and followed by melt-spinning to prepare the alloy ribbons. The tangent speed of the $\mathrm{Cu}$ roller is $45 \mathrm{~m} \mathrm{~s}^{-1}$. The obtained alloy ribbon is $\sim 20 \mu \mathrm{m}$ thick and $\sim 3 \mathrm{~mm}$ wide. The alloy ribbons were etched in a $1 \mathrm{M}\left(\mathrm{NH}_{4}\right)_{2} \mathrm{SO}_{4}$ solution at $50{ }^{\circ} \mathrm{C}$ for $12 \mathrm{~h}$ to prepare the nanoporous alloys. After dealloying, the ribbons were rinsed thoroughly with deionized water and ethanol to remove chemical residuals in nanoporous channels and used as binder-free electrode for both HER/OER and overall water-splitting measurements. The free-standing nanoporous alloys were also milled into powder $(4.0 \mathrm{mg})$ and mixed with carbon $(3.0 \mathrm{mg})$, isopropyl alcohol $(300 \mu \mathrm{L})$ and Nafion solution (100 $\mu \mathrm{L}, 0.5 \mathrm{wt} . \%$ ). After sonification mixing for $30 \mathrm{~min}$, the mixture suspension $(4 \mu \mathrm{L})$ was coated on a glassy carbon electrode (GCE) for the ORR test. Purchased Pt/C (20 wt.\%, johnson-matthey) and $\mathrm{IrO}_{2}$ (Aladdin) catalysts were studied for reference.

\section{Materials characterization}

The microstructures of dealloyed HEAs were characterized by two transmission electron microscopes (JEOL ARM 200F). One is equipped with a cold emission gun and an aberration corrector for the probe-forming lens system to obtain the atomic 
resolution STEM images, another one with an aberration corrector for the objective lens system to obtain high quality HRTEM images and SAED patterns operated under an acceleration voltage of $200 \mathrm{KeV}$. The probe convergence semi-angle was approximately $30 \mathrm{mrad}$. Selected area electron diffraction (SAED) patterns were acquired at a camera length of $20 \mathrm{~mm}$. High-angle annular dark field scanning TEM (HAADF-STEM) images were recorded using an annular-type detector with collection semi-angle $\sim 100-269$ mrad. STEM-EDS spectra and corresponding elemental mapping were acquired at nano-scale.

Scanning electron microscope (SEM, HITACHI S-4700) also equipped with EDS, an X-ray diffraction (XRD) diffractometer using $\mathrm{Cu} \mathrm{K \alpha}$ radiation (Rigaku D/Max 2500), and an X-ray photoelectron spectroscopy (XPS, ESCALAB 250) were also used to study the structure and composition. An electrochemical workstation (CHI-760E) was used for electrochemical studies.

\section{Electrochemical measurements}

A standard three-electrode setup was utilized for HER, OER, and ORR tests, which included a free-standing nanoporous HEA or modified GCE as the working electrode, a carbon rod as counter electrode and an $\mathrm{Ag} / \mathrm{AgCl}$ electrode as reference electrode. The HER test was carried out in a $\mathrm{H}_{2}$ saturated $1.0 \mathrm{M} \mathrm{KOH}$ solution. Both the OER and ORR tests were conducted under pure $\mathrm{O}_{2}$ saturation to ensure the $\mathrm{O}_{2} / \mathrm{H}_{2} \mathrm{O}$ equilibrium at $1.23 \mathrm{~V}$ vs RHE. Linear scan voltammograms and cyclic voltammetry were conducted with a scan rate of $5 \mathrm{mV} \mathrm{s}^{-1}$. The current density was normalized to the geometrical area of the binder-free electrode or the modified GCE. The measured potentials vs 
$\mathrm{Ag} / \mathrm{AgCl}$ were converted to a reversible hydrogen electrode (RHE) scale according to the Nernst equation $\left(\mathrm{E}_{\mathrm{RHE}}=\mathrm{E}_{\mathrm{Ag} / \mathrm{AgCl}}+0.059 \mathrm{pH}+0.197\right)$; the overpotential $(\eta)$ was calculated according to the following formula: $\eta(\mathrm{V})=\mathrm{E}_{\mathrm{RHE}}-1.23 \mathrm{~V}$. The loading amounts of different catalysts used in electrochemical tests are shown in Table S5. For the overall water splitting, two same binder-free nanoporous HEAs were used as symmetry anode and cathode.

\section{Battery tests}

The Zn-air batteries were assembled with the catalyst modified carbon cloth (catalyst loading: $\left.\sim 0.5 \mathrm{mg} \mathrm{cm}^{-2}\right)$ as the air cathode, a $\mathrm{Zn}$ foil $(0.10 \mathrm{~mm}$ in thickness $)$ as the anode, and a mixed aqueous solution of zinc acetate $(0.2 \mathrm{M})$ and potassium hydroxide $(6.0 \mathrm{M})$ solution as the electrolyte. $\mathrm{A} \mathrm{Pt} / \mathrm{C}$ - $\mathrm{IrO}_{2}$-based battery was assembled by the same way for reference. During the long-term battery test, the electrolyte was changed after every $100 \mathrm{~h}$.

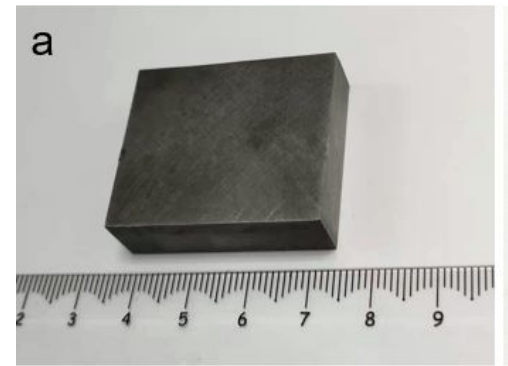

b

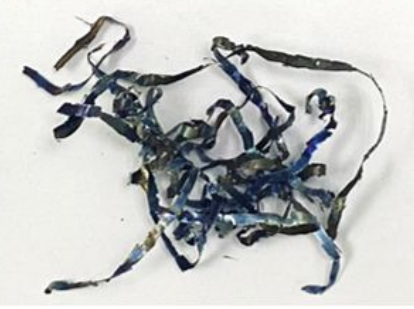

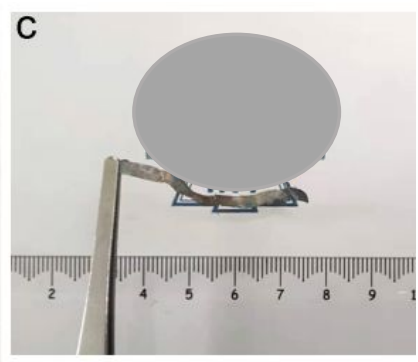

Figure S1. Photographs of the prepared 12-component bulk ingot (a), the meltspinned 12-component alloy ribbons (b) and the dealloyed free-standing np-12 (c). 
a

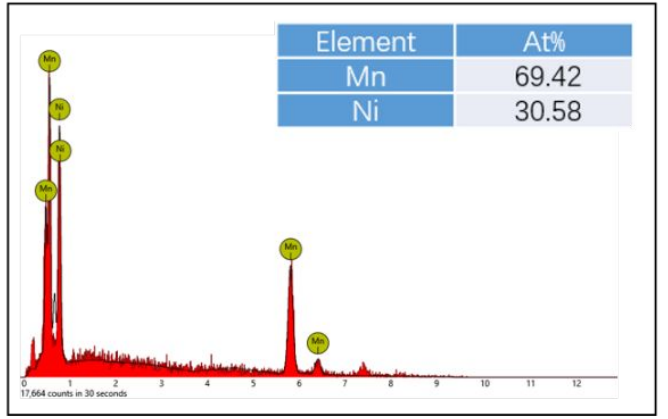

C

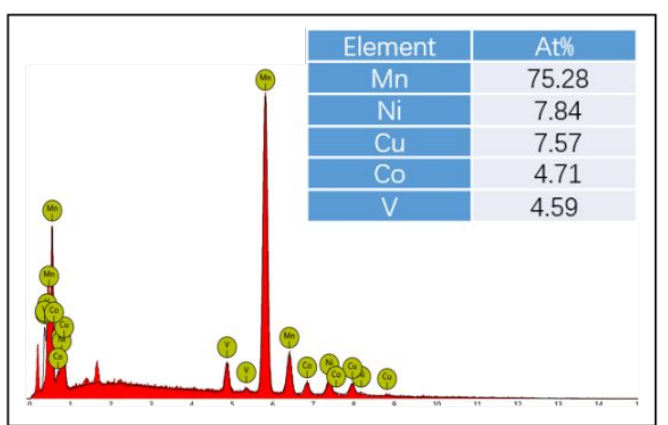

b

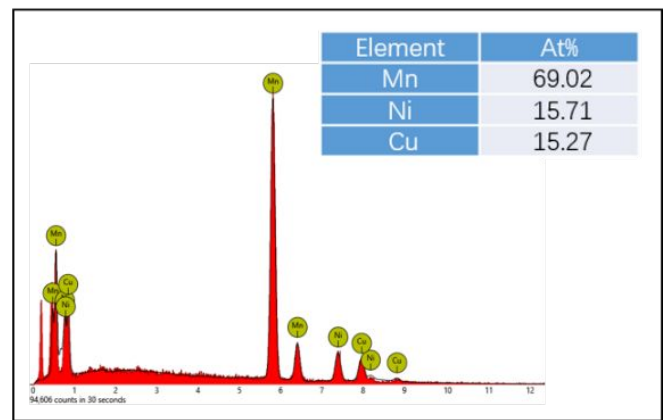

d

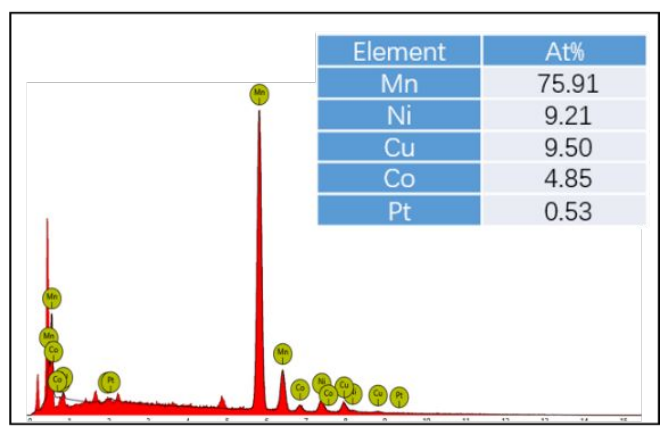

e
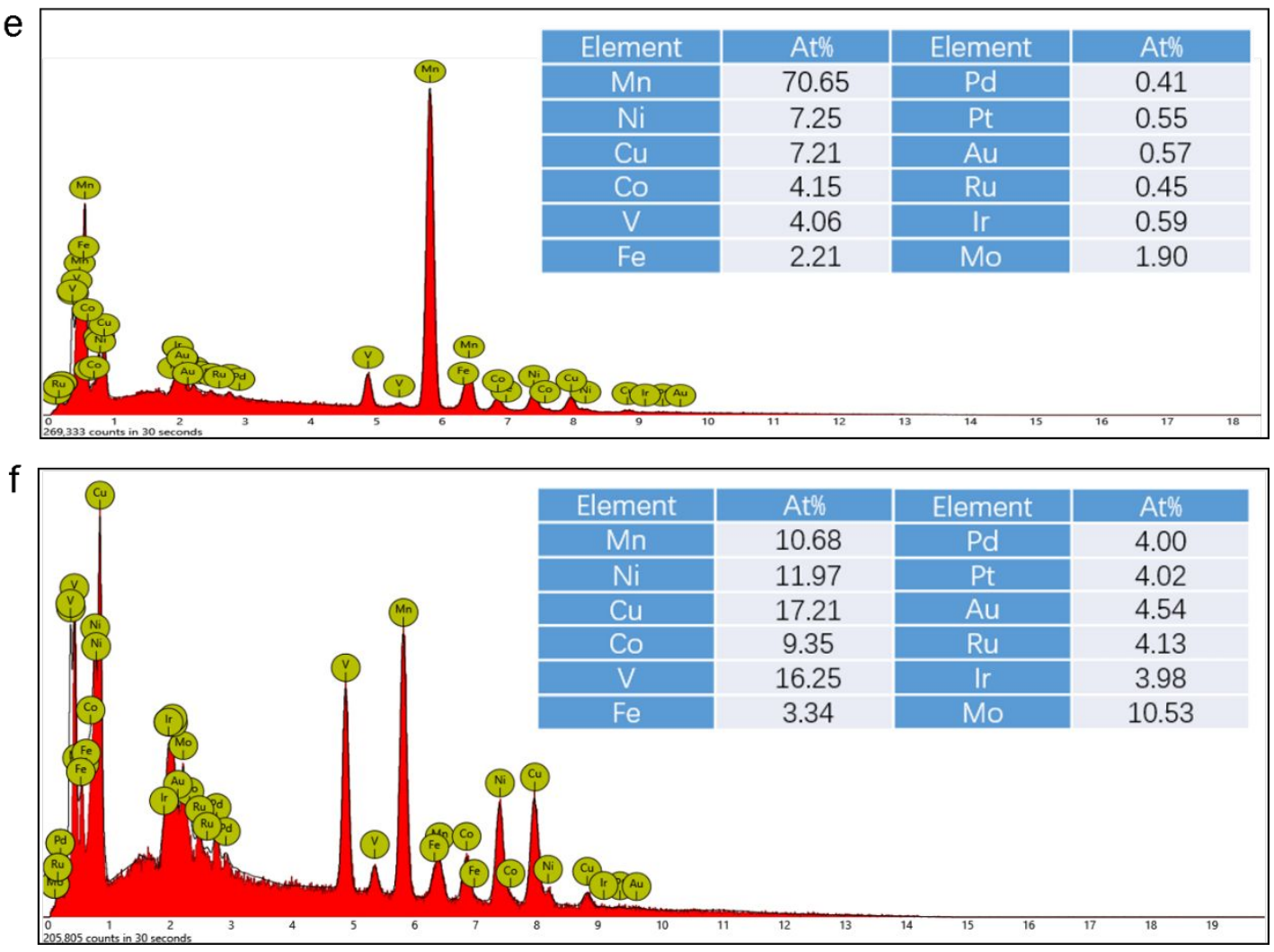
g

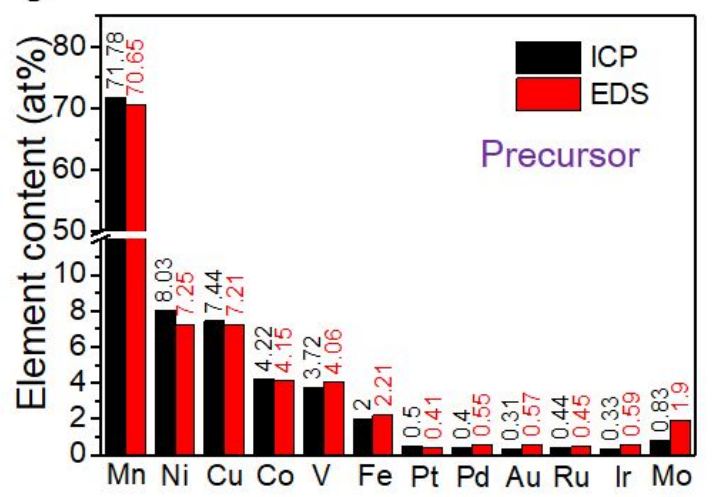

$\mathrm{h}$

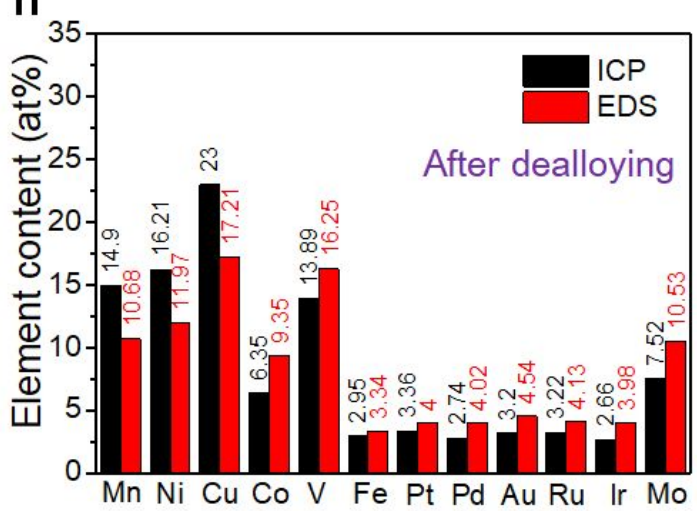

Figure S2. EDS spectra of the $\mathrm{MnNi}$ alloy (a), $\mathrm{MnNiCu}$ alloy (b), $\mathrm{MnNiCuCoV}$ alloy (c), MnNiCuCoPt alloy (d) and the 12-component MnNiCuCoVFeMoPdPtAuRuIr precursors (e). EDS spectra of the dealloyed np-12 (f). Comparison of the ICP and EDS results of the 12-component sample before ( $\mathrm{g}$ ) and after dealloying (h).

Based on the EDS results, the oxygen content in the np-12 is around 35 at.\%, suggesting the oxidized states of these surface non-noble metals. 

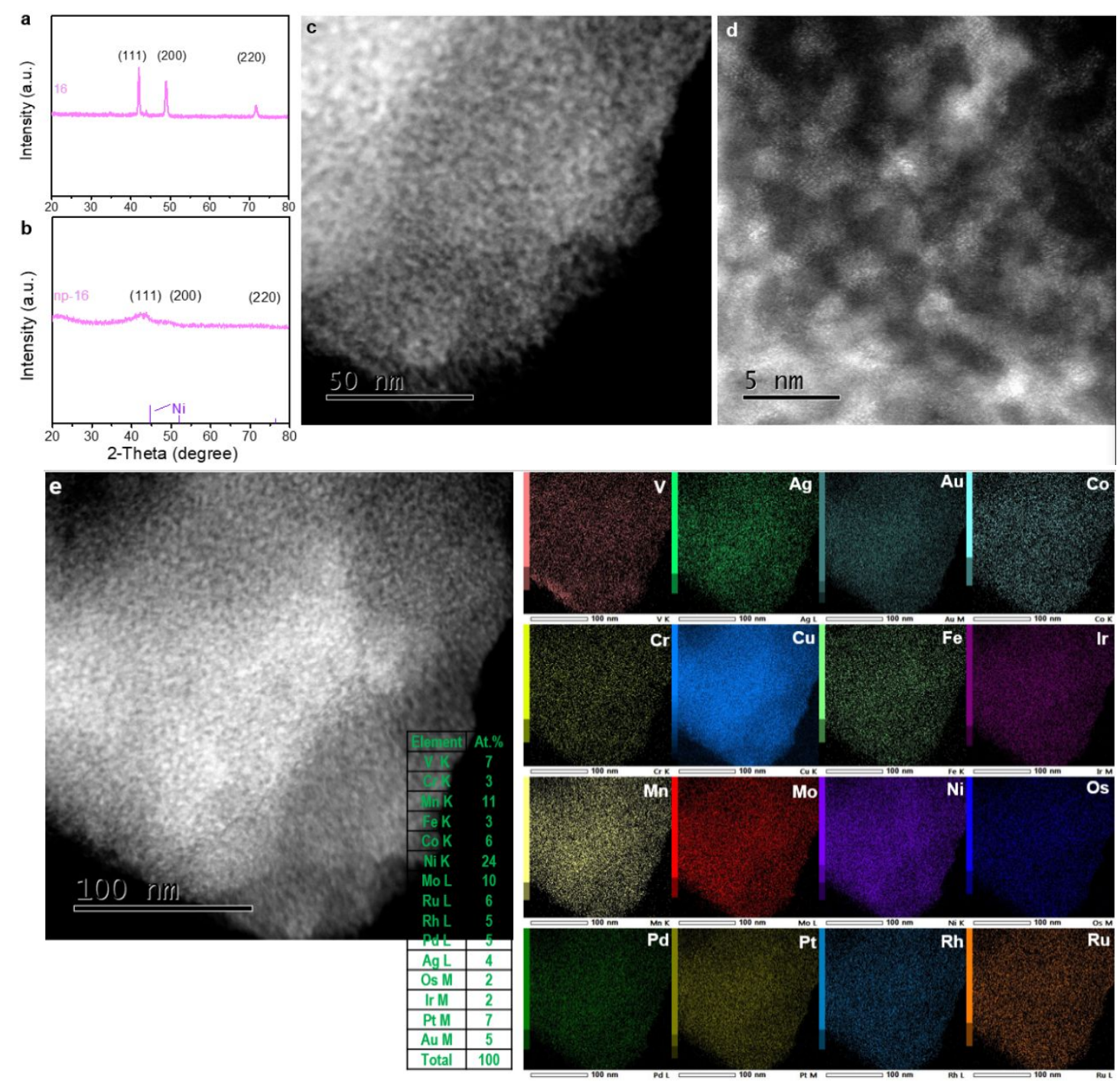

Figure S3. XRD patterns of the 16-component

MnNiCuCoVFeCrMoPdPtAuAgRuIrRhOs alloy before (a) and after (b) dealloying. STEM images with different magnification (c, d) and STEM-EDS mapping (e) of the dealloyed $\mathrm{np}-16$. Inset in (e) shows the atomic ratio of the each element without $\mathrm{Cu}$ due to the interference from $\mathrm{Cu}$ grid holder. 


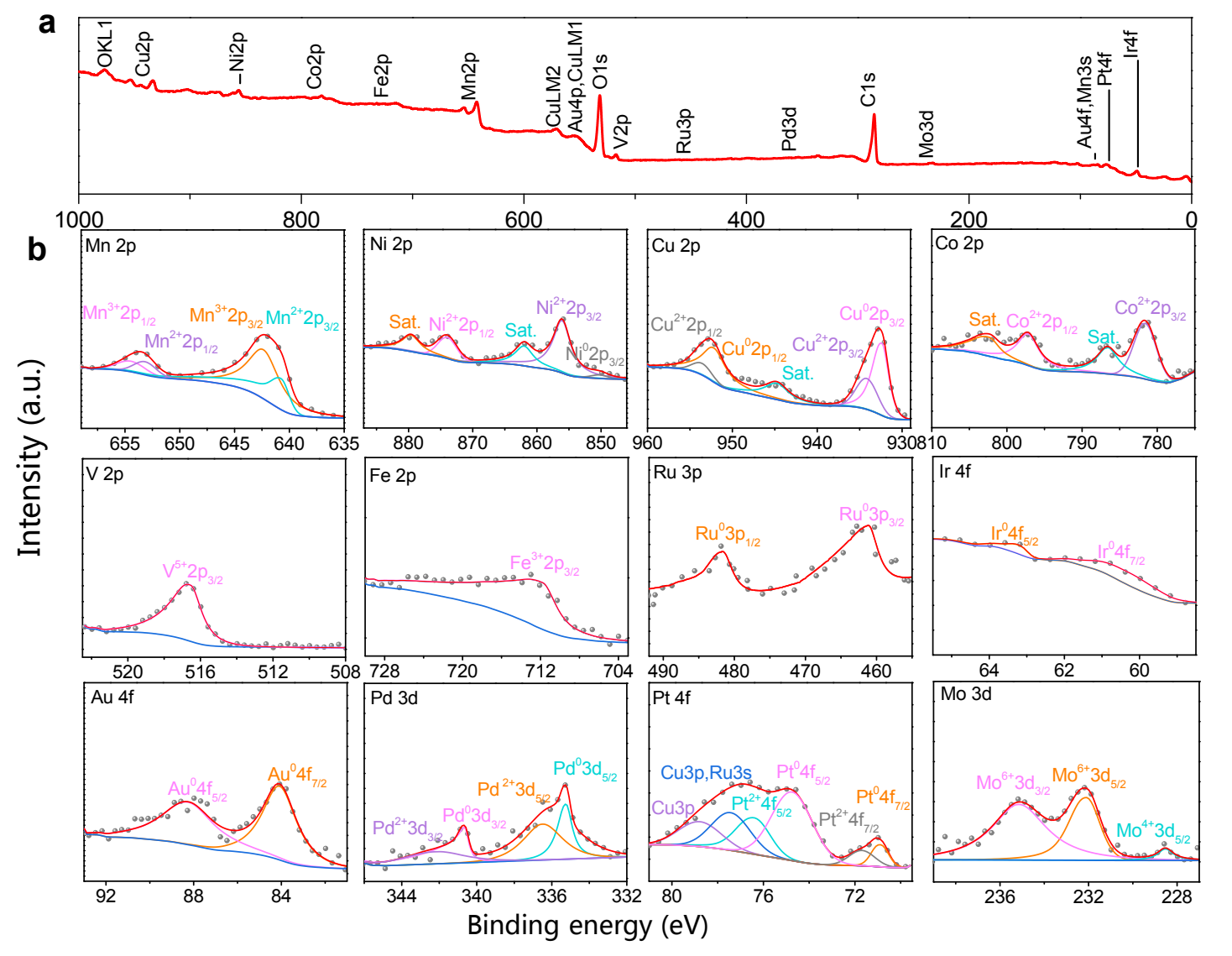

Figure S4. Complete XPS spectrum showing the existence of 12 elements of the np12 in the $0-1000 \mathrm{eV}$ region (a). XPS spectra and deconvoluted states for each element in $\mathrm{np}-12$ (b).
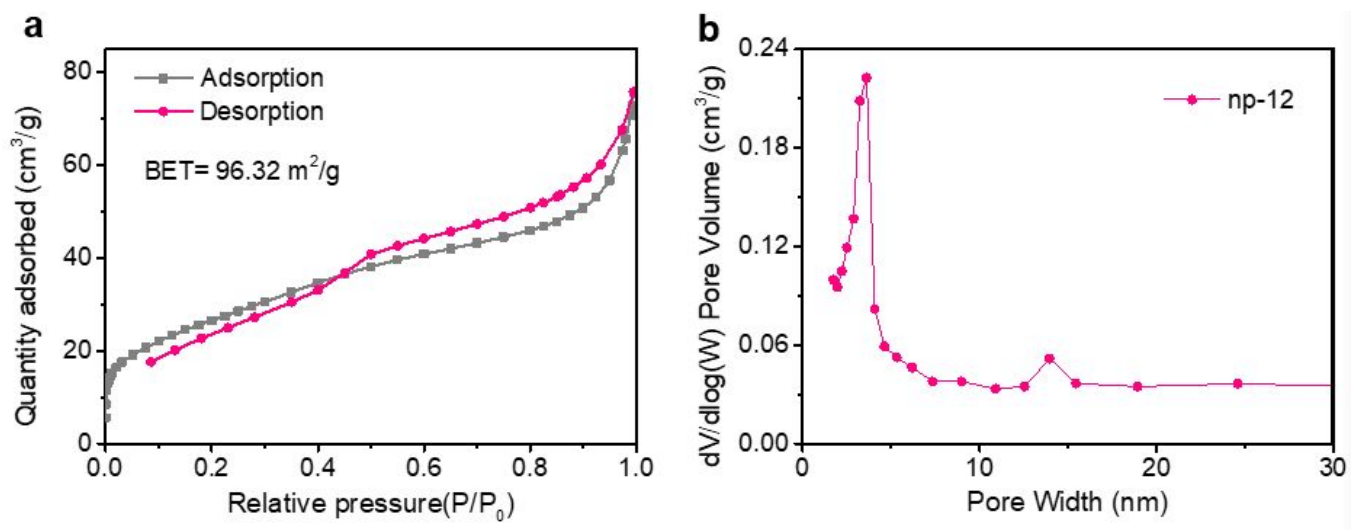

Figure S5. $\mathrm{N}_{2}$ adsorption-desorption isotherms (a) and pore size distribution (b) of the dealloyed np-12. 

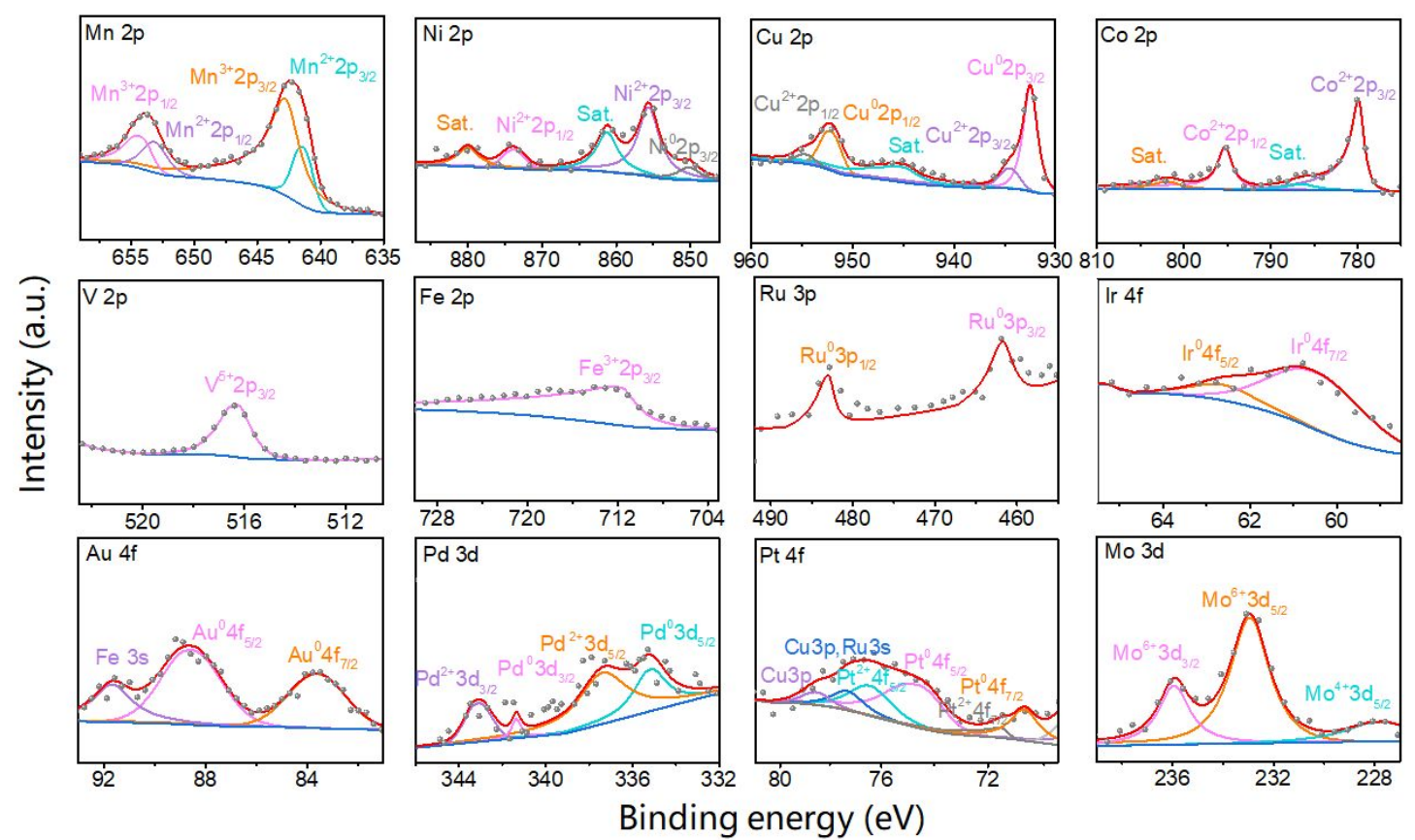

Figure S6. XPS spectra for each element in np-12 after the OER i-t test.

Similar to the XPS result of the np-12 before OER reaction, the non-noble elements such as $\mathrm{Mn}, \mathrm{Ni}, \mathrm{Co}, \mathrm{V}, \mathrm{Fe}, \mathrm{Mo}$ are still in their high oxidation states. In comparison, we found that after the reaction, more oxidized $\mathrm{Pd}\left(\mathrm{Pd}^{2+}\right)$ appeared. We also found a clear shift of $\mathrm{Au}^{0} 4 \mathrm{f}_{7 / 2}$ peak to lower binding energy and shift of $\mathrm{Mo}^{6+} 3 \mathrm{~d}_{5 / 2}$ to higher binding energy. These results suggest the changed surface electronic structure by the applied high potential during the OER process.

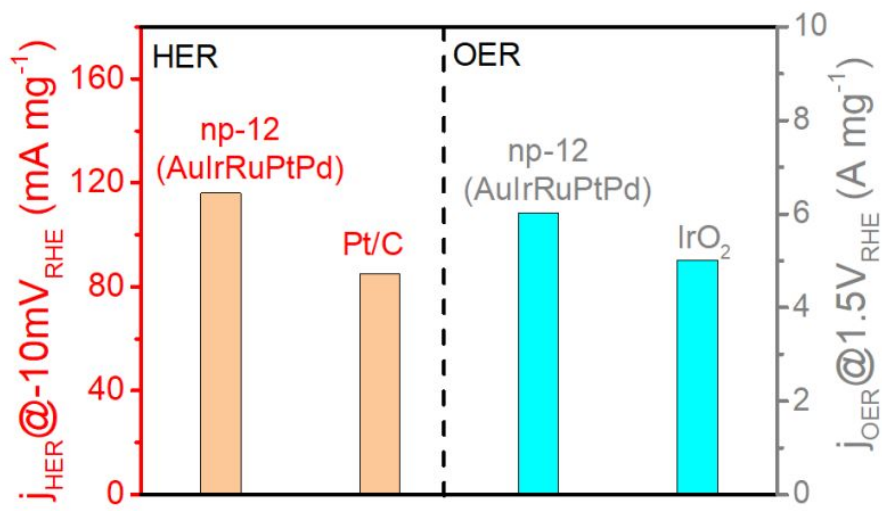

Figure S7. Comparison of the noble metal-based mass activity of the np-12 and commercial catalysts. For the np-12, all the five noble elements are considered. 

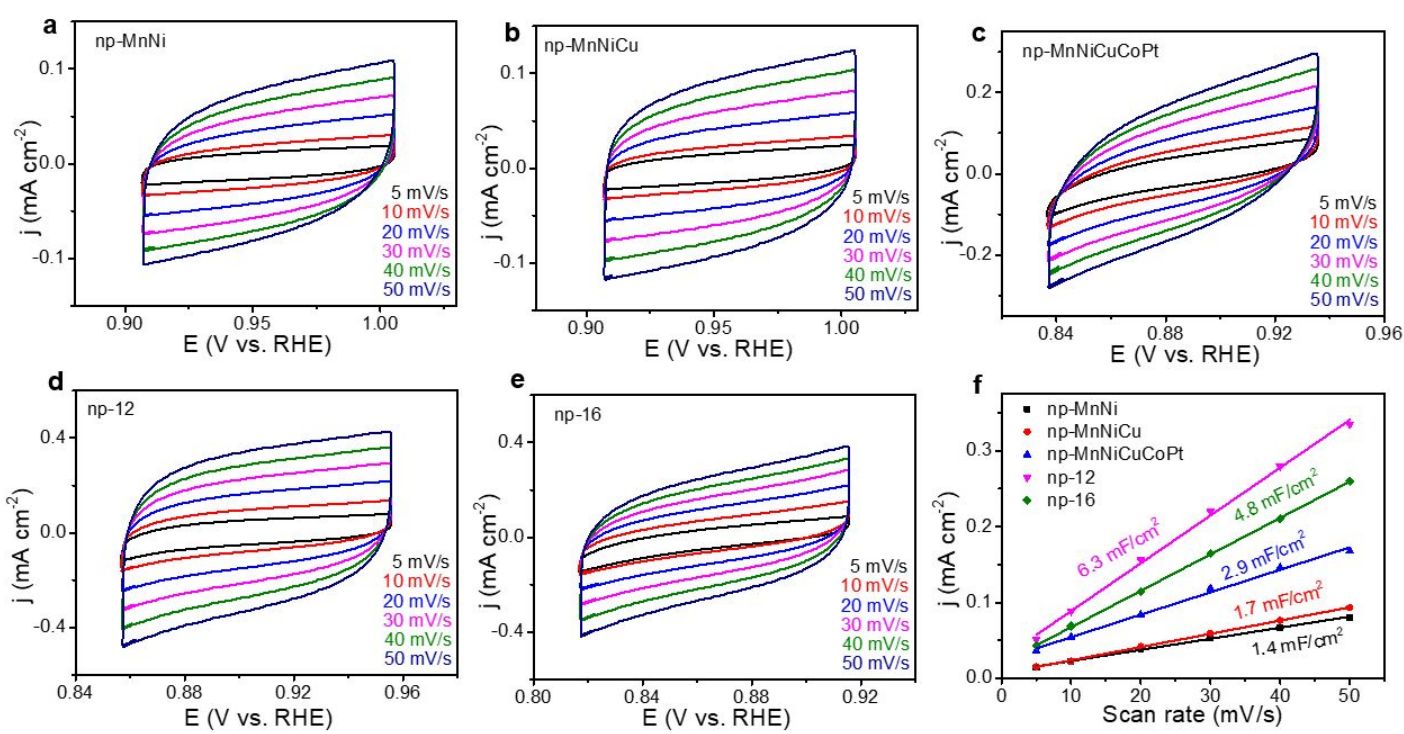

Figure S8. CV curves of these catalysts in $1.0 \mathrm{M} \mathrm{KOH}$ solution showing the doublelayer capacitance without electrochemical reactions (a-e). The difference in current density $(\Delta \mathrm{J}=\mathrm{Ja}-\mathrm{Jc})$ plotted against scan rate $(\mathrm{f})$ and fitted to a linear regression for the estimation of capacitance (f).
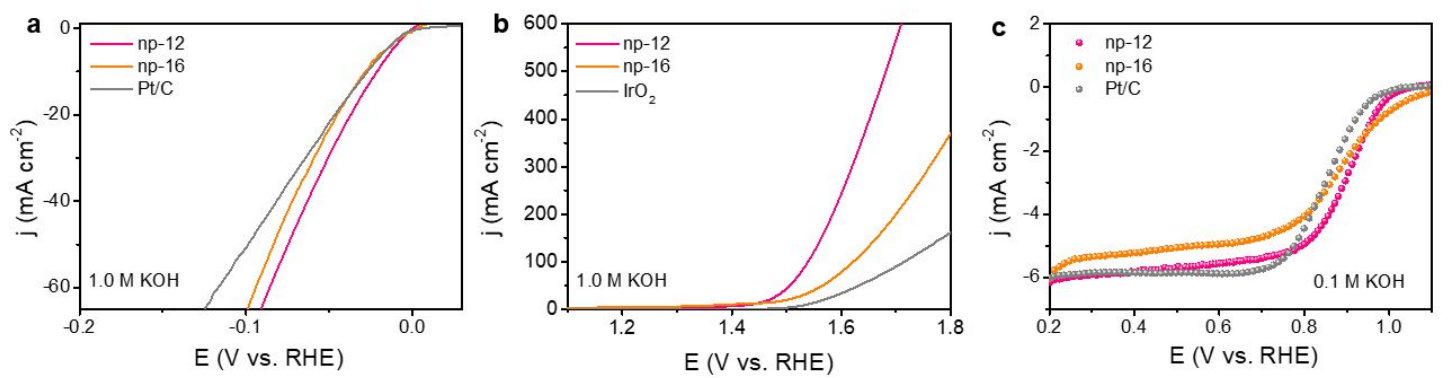

Figure S9. HER (a), OER (b) and ORR (c) polarization curves of the np-12, np-16 and commercial catalysts.

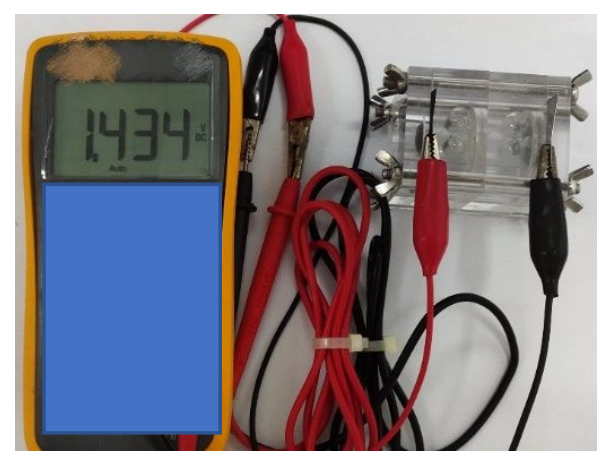

Figure S10. Open-circuit voltage of the $\mathrm{Pt} / \mathrm{C}-\mathrm{IrO}_{2}$-based $\mathrm{Zn}$-air battery. 

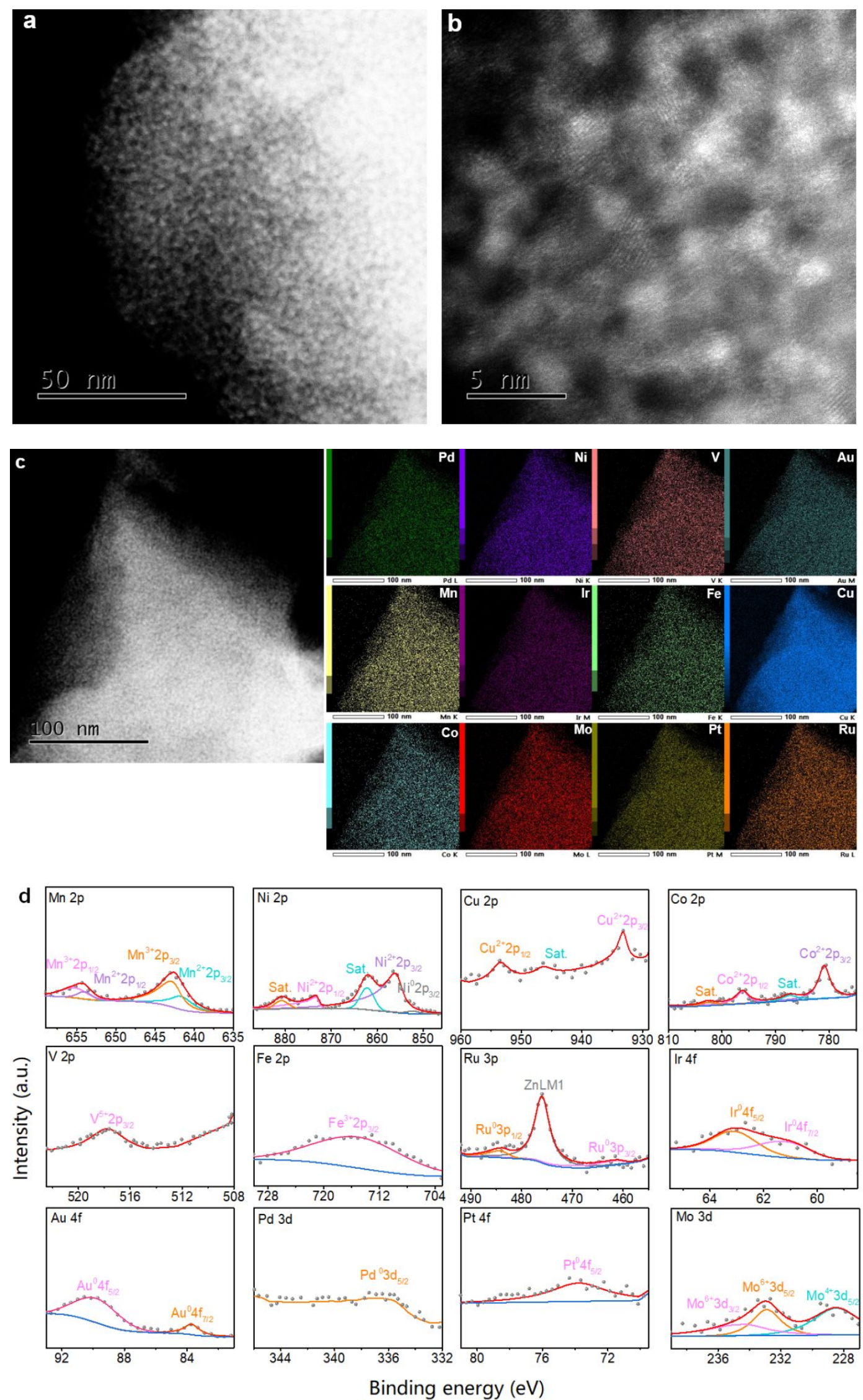

Figure S11. STEM (a, b) , STEM-EDS mapping (c) and XPS spectra for each element (d) of the np-12 after battery cycling test. 
Table S1. The HER, OER bifunctional catalytic performance of np-12 compared with literature data.

$\begin{array}{ccccccc}\text { Catalysts } & \text { HER } & \text { Tafel } & \text { OER } & \text { Tafel } \\ & \eta 10(\mathrm{mV}) & (\mathrm{mV} / \mathrm{dec}) & \eta 10(\mathrm{mV}) & (\mathrm{mV} / \mathrm{dec}) & \text { Solution } & \text { Refs. }\end{array}$

np-12

21

np-AlNiCoRuMo

24.5

31.4

245

$\mathrm{Rh}_{6} \mathrm{Cu}_{1} / \mathrm{C}$

36

353

66

320

Co/CNFs (1000)

190

$\mathrm{Ru}-\mathrm{CoV}-$

$\mathrm{LDH} / \mathrm{NF}$

$\mathrm{Ru} / \mathrm{N}-\mathrm{BP} 2000-4$

wt $\%$

20

not given

285

not given

$1.0 \mathrm{M}$

$\mathrm{KOH}$

$1.0 \mathrm{M}$
$\mathrm{KOH}$

Adv. Mater. 2019,

79

81.2

$1.0 \mathrm{M}$

$\mathrm{KOH}$

ChemSusChem

2021, 14, 730.

$$
\mathrm{Pt}_{62} \mathrm{Co}_{23} / \mathrm{Ir}_{15}
$$

FBNWs/C

24

not given

308

not given

203

NiVIr-LDH

41

39.5

86
78
180

ACS Materials

Lett. 2020, 2, 12, 1698-1706

ACS Appl. Mater.

Interfaces 2020,

12, 9, 10299

10306

31,1808043

Co/MMCs

78
ACS Energy Lett. 2019, 4, 8, 18231829

ChemElectroChem 2019, 6, 4522
$1.0 \mathrm{M}$

$\mathrm{KOH}$ 
Table S2. The overall water-splitting performance of the np-12 compared with literature data.

\begin{tabular}{|c|c|c|c|c|c|}
\hline Catalysts & Electrolyte & $\begin{array}{c}\text { Current } \\
\text { density } \\
\left(\mathrm{mA} \mathrm{cm}^{-2}\right)\end{array}$ & Potential (V) & $\begin{array}{l}\text { Mass loading } \\
\left(\mathrm{mg} \mathrm{cm}^{-2}\right)\end{array}$ & Refs. \\
\hline np-12 & $1.0 \mathrm{M} \mathrm{KOH}$ & $\begin{array}{c}10 \\
200\end{array}$ & $\begin{array}{l}1.49 \\
1.82\end{array}$ & $\begin{array}{c}0.006 \\
\text { (AuIrRuPtPd) }\end{array}$ & This work \\
\hline np-AlNiCoRuMo & $1.0 \mathrm{M} \mathrm{KOH}$ & 10 & 1.5 & $0.048(\mathrm{Ru})$ & $\begin{array}{c}\text { ACS Materials Lett. 2020, } \\
\text { 2, 12, 1698-1706 }\end{array}$ \\
\hline $\mathrm{Rh}_{6} \mathrm{Cu}_{1} / \mathrm{C}$ & $1.0 \mathrm{M} \mathrm{KOH}$ & $\begin{array}{c}10 \\
120\end{array}$ & $\begin{array}{l}1.55 \\
1.96\end{array}$ & 0.282 & $\begin{array}{c}\text { ACS Appl. Mater. } \\
\text { Interfaces 2020, 12, 9, } \\
\text { 10299-10306 }\end{array}$ \\
\hline $\mathrm{Co} / \mathrm{CNFs}(1000)$ & $0.1 \mathrm{M} \mathrm{KOH}$ & 10 & 1.69 & 0.3 & $\begin{array}{c}\text { Adv. Mater. 2019, 31, } \\
1808043\end{array}$ \\
\hline $\mathrm{Ru}-\mathrm{CoV}-\mathrm{LDH} / \mathrm{NF}$ & $1.0 \mathrm{M} \mathrm{KOH}$ & 10 & 1.5 & not given & $\begin{array}{c}\text { ChemSusChem 2021, 14, } \\
730\end{array}$ \\
\hline $\begin{array}{c}\mathrm{Ru} / \mathrm{N}-\mathrm{BP} 2000-4 \\
\mathrm{wt} \%\end{array}$ & $1.0 \mathrm{M} \mathrm{KOH}$ & $\begin{array}{l}10 \\
20\end{array}$ & $\begin{array}{c}1.53 \\
\sim 1.59\end{array}$ & 0.285 & $\begin{array}{c}\text { ChemCatChem 2019, 11, } \\
4327\end{array}$ \\
\hline $\begin{array}{l}\mathrm{Pt}_{62} \mathrm{Co}_{23} / \mathrm{Ir}_{15} \\
\text { FBNWs/C }\end{array}$ & $0.1 \mathrm{M} \mathrm{KOH}$ & 10 & 1.65 & 0.04 (PtIr) & $\begin{array}{c}\text { Chem. Mater. 2019, 31, } 19, \\
\text { 8136-8144 }\end{array}$ \\
\hline NiVIr-LDH & $1.0 \mathrm{M} \mathrm{KOH}$ & 10 & 1.49 & 1.7 & $\begin{array}{c}\text { ACS Energy Lett. 2019, 4, } \\
\text { 8, 1823-1829 }\end{array}$ \\
\hline $\mathrm{Co} / \mathrm{MMCs}$ & $1.0 \mathrm{M} \mathrm{KOH}$ & 10 & 1.66 & 0.22 & $\begin{array}{c}\text { ChemElectroChem 2019, } 6 \text {, } \\
4522\end{array}$ \\
\hline
\end{tabular}


Table S3. ORR performance in alkaline solution of np-12 compared with reported noble metal-based catalysts.

\begin{tabular}{|c|c|c|c|c|}
\hline Catalysts & Electrolyte & $\begin{array}{c}\mathrm{E}_{1 / 2} \\
\text { (V vs. RHE) }\end{array}$ & $\begin{array}{l}\text { Mass loading } \\
\left(\mathrm{mg} \mathrm{cm}^{-2}\right)\end{array}$ & Refs. \\
\hline np-12 & $0.1 \mathrm{M} \mathrm{KOH}$ & 0.90 & $\begin{array}{c}0.006 \\
\text { (AuIrRuPtPd) }\end{array}$ & This work \\
\hline $\mathrm{Pt} / \mathrm{C}$ & $0.1 \mathrm{M} \mathrm{KOH}$ & 0.85 & $0.007(\mathrm{Pt})$ & This work \\
\hline np-AlNiCoRuMo & $0.1 \mathrm{M} \mathrm{KOH}$ & 0.875 & $0.048(\mathrm{Ru})$ & $\begin{array}{c}\text { ACS Materials Lett. } \\
2020,2,12,1698-1706 .\end{array}$ \\
\hline PdMo bimetallene/C & $0.1 \mathrm{M} \mathrm{KOH}$ & 0.95 & 0.0075 & Nature 2019, 574, 81-85. \\
\hline $\mathrm{PtCo}-3 \mathrm{NC}$ & $1.0 \mathrm{M} \mathrm{KOH}$ & 0.86 & 0.0245 & $\begin{array}{c}\text { Appl. Catal. B-Environ. } \\
\text { 2018, 236, } 413 .\end{array}$ \\
\hline PdCo-300 & $0.1 \mathrm{M} \mathrm{KOH}$ & 0.83 & 0.455 & $\begin{array}{c}\text { Appl. Catal. B-Environ. } \\
2019,243,175 .\end{array}$ \\
\hline Ag NP/NiRu-LDHs & $0.1 \mathrm{M} \mathrm{KOH}$ & 0.78 & 0.070 & $\begin{array}{c}\text { ACS Catal. } \\
2019,9,117-129 .\end{array}$ \\
\hline Pd-Ru@NG & $0.1 \mathrm{M} \mathrm{KOH}$ & 0.8 & 0.38 & $\begin{array}{l}\text { Chem. Commun., } \\
\text { 2019, 55, } 13928 .\end{array}$ \\
\hline
\end{tabular}


Table S4. Comparison of the liquid Zn-air battery performance with literature data.

\begin{tabular}{|c|c|c|c|c|}
\hline Catalysts & $\begin{array}{l}\text { Open circuit } \\
\text { potential }(\mathrm{V})\end{array}$ & $\begin{array}{l}\text { Peak power } \\
\text { density } \\
\left.(\mathrm{mW} \mathrm{cm})^{-2}\right)\end{array}$ & Stability & Refs. \\
\hline np-12 & 1.50 & 122 & $\begin{array}{c}240 \text { h240 cycles@10 } \\
\text { mA cm-2 }\end{array}$ & This work \\
\hline LIG-MnNiFe-1 & 1.42 & 98.9 & $\begin{array}{c}350 \text { h2100 cycles@10 } \\
\text { mA cm-2 }\end{array}$ & $\begin{array}{c}\text { ACS Appl. Energy } \\
\text { Mater. 2019, 2, 2, } \\
1460-1468\end{array}$ \\
\hline NCNF-1000 & 1.48 & 185 & $\begin{array}{c}83.3 \text { h } 500 \text { cycles@10 } \\
\text { mA cm-2 }\end{array}$ & $\begin{array}{c}\text { Adv. Mater. 2016, } 28 \\
\text { 3000-3006 }\end{array}$ \\
\hline $\mathrm{Co} 4 \mathrm{~N} / \mathrm{CNW} / \mathrm{CC}$ & 1.40 & 174 & $136 \mathrm{~h} @ 10 \mathrm{~mA} \mathrm{~cm}{ }^{-2}$ & $\begin{array}{c}\text { J. Am. Chem. Soc. } \\
\text { 2016, 138, } \\
\text { 10226-10231 }\end{array}$ \\
\hline N-GCNT/FeCo-3 & 1.48 & 89.3 & $9 \mathrm{~h} @ 20 \mathrm{~mA} \mathrm{~cm}{ }^{-2}$ & $\begin{array}{c}\text { Adv. Energy Mater. } \\
2017,7,1602420\end{array}$ \\
\hline NPHG-8 & 1.32 & 30 & $\begin{array}{c}176 \text { h176 cycles@2 } \\
\text { mA cm }{ }^{-2}\end{array}$ & $\begin{array}{c}\text { ChemElectrochem } \\
2018,5,1811\end{array}$ \\
\hline $\mathrm{CoIn}_{2} \mathrm{~S}_{4} / \mathrm{S}-\mathrm{rGO}$ & 1.42 & 133 & $\begin{array}{c}50 \text { h150 cycles@10 } \\
\text { mA cm }{ }^{-2}\end{array}$ & $\begin{array}{c}\text { Adv. Energy Mater. } \\
2018,8,1802263 .\end{array}$ \\
\hline Co-N, B-CSs & 1.43 & 100.4 & $14 \mathrm{~h} @ 5 \mathrm{~mA} \mathrm{~cm}{ }^{-2}$ & $\begin{array}{c}\text { ACS Nano } 2018 \\
\text { 12,1894-1901. }\end{array}$ \\
\hline C-MOF-C2-900 & -- & 105 & $\begin{array}{c}120 \text { h } 360 \text { cycles@2 } \\
\text { mA cm }{ }^{-2}\end{array}$ & $\begin{array}{c}\text { Adv. Mater. 2018, 30, } \\
1705431\end{array}$ \\
\hline NGM-Co & 1.44 & 152 & $60 \mathrm{~h} @ 2 \mathrm{~mA} \mathrm{cm-2}$ & $\begin{array}{c}\text { Adv. Mater. 2017, 29, } \\
1703185\end{array}$ \\
\hline SilkNC/KB & 1.426 & 91.2 & $33 \mathrm{~h} @ 10 \mathrm{~mA} \mathrm{~cm}-2$ & $\begin{array}{c}\text { Chem. Mater. 2019, 31, } \\
\text { 3.1023-1029 }\end{array}$ \\
\hline
\end{tabular}


$\mathrm{AlFeCoNiCr}$

1.55

Mo-N/C@ $\mathrm{MoS}_{2}$

1.342

CoOx@NGCR

$\mathrm{Pt} / \mathrm{C}-\mathrm{IrO}_{2}$

$\mathrm{CoMn}_{1} \mathrm{Cr}_{1} \mathrm{O}_{4}$

1.37

$\mathrm{Fe}_{0.5} \mathrm{Co}_{0.5} \mathrm{O}_{\mathrm{x}} / \mathrm{NrGO}$

$\mathrm{FeCo} / \mathrm{Se}-\mathrm{CNT}$

1.54

1.45

CNTs/rGO

$\mathrm{Co}-\mathrm{TiO}_{2}$

$\mathrm{N}-\mathrm{CoSe}_{2} / 3 \mathrm{D} \mathrm{Ti}_{3} \mathrm{C}_{2} \mathrm{~T}_{\mathrm{x}}$

1.43

142

136

131.4

173.4

200 2019, 1, 4, 432-439

Adv. Mater. 2017, 29 , 1702327
$440 \mathrm{~h} @ 5$ mA cm ${ }^{-2}$

Nanoscale, 2019,11, 21943-21952

J. Mater. Chem. A, 2016,4, 7841-7847

ACS Materials Lett.

2017, 27, 1702300

ACS Sustainable Chem.

Eng. 2018, 6,

15811-15821

Chem. Mater. 2021, 33,

5, 1771-1780

J. Power Sources. 2020, 479, 229099

ACS Appl. Mater. 29448-29456

Nano Lett. 2021, 21, 5, 2255-2264

$166 \mathrm{~h} @ 10 \mathrm{~mA} \mathrm{~cm}{ }^{-2}$ 
Ni-Fe-MoN NTs

NC-Co SA

NS-CC

1.247

47

$40 \mathrm{~h} @ 20 \mathrm{~mA} \mathrm{~cm}{ }^{-2}$

600 h 300 cycles@10

$\mathrm{mA} \mathrm{cm}{ }^{-2}$

$\mathrm{NiCo} / \mathrm{PFC}$ aerogels

$-$
Adv. Energy Mater.

2018, 8, 1802327

ACS Catal. 2018, 8, 8961-8969

Adv. Sci. 2018, 5, 1800760

Nano Lett. 2016, 16, 10, 6516-6522 ( $\mathrm{cm}^{-2}$

(a) 
Table S5. Noble metal loading amounts of different electrocatalysts for different tests.

\begin{tabular}{|c|c|c|c|c|c|}
\hline Materials & $\begin{array}{c}\text { HER } \\
\left(\mathrm{mg} \mathrm{cm}^{-2}\right)\end{array}$ & $\begin{array}{c}\text { OER } \\
\left(\mathrm{mg} \mathrm{cm}^{-2}\right)\end{array}$ & $\begin{array}{c}\text { Over-all water } \\
\text { splitting } \\
\left(\mathrm{mg} \mathrm{cm}^{-2}\right)\end{array}$ & $\begin{array}{c}\text { ORR } \\
\left(\mathrm{mg} \mathrm{cm}^{-2}\right)\end{array}$ & $\begin{array}{c}\text { Zn-air battery } \\
\left(\mathrm{mg} \mathrm{cm}^{-2}\right)\end{array}$ \\
\hline $\begin{array}{c}\text { np-12 } \\
\text { (AuIrRuPtPd) }\end{array}$ & 0.037 & 0.037 & 0.037 & 0.006 & 0.045 \\
\hline $\begin{array}{c}\text { np-16 } \\
\text { (PdPtAuRuIrAgRhOs) }\end{array}$ & 0.044 & 0.044 & 0.044 & 0.007 & 0.055 \\
\hline $\mathrm{IrO}_{2}$ & & 0.007 & 0.007 & -- & 0.025 \\
\hline $20 \mathrm{wt} . \% \mathrm{Pt} / \mathrm{C}$ & 0.007 & -- & 0.007 & 0.007 & 0.025 \\
\hline
\end{tabular}

